\title{
Evaluation of research ethics committees in Turkey
}

Berna Arda Ankara University, Ankara, Turkey

\begin{abstract}
In Turkey, there was no legal regulation of research on human beings until 1993. In that year "the amendment relating to drug researches" was issued. The main objectives of the regulation are to establish a central ethics committee and local ethics committees, and to provide administrative control. There are no compulsory clinical ethics lectures in the medical curriculum, so it is also proposed that research ethics committees (RECs) play a central educational role by helping physicians to be aware of moral problems and by contributing to the training of research teams.

(Fournal of Medical Ethics 2000;26:459-461)
\end{abstract}

Keywords: Medical ethics education; ethics committees in Turkey; good clinical practice; informed consent; respiratory distress syndrome

\section{Introduction}

Good clinical practice (GCP) is a new concept in my country, which can serve as a guide to physicians in routine medical practice. Its first aim is to determine the standards of competence, care and conduct for "good" practice in terms of ethics. This concept is extremely important in developing countries such as Turkey, because patient rights is a new idea and such rights are not commonly recognised, and also because there are many problems in the health systems in these countries. Another problem is medical ethics education: there is no standard by which to organise its introduction and content within Turkey.

It must be stressed that the teaching of medical ethics poses particular problems because of the specific nature of the subject. As generally accepted, all the basic concepts related to ethics and morals, such as duty, responsibility, obligation, rights and so on, are attached to various schools of philosophy and these may differ in different cultures. Indeed, these concepts represent the related basic idealisations of philosophers, intellectuals, and societies on human conduct in various contexts.

In this paper, medical ethics education, and especially the teaching of clinical ethics are evaluated in this country in the light of recent legislation and the general educational approach.

\section{Medical ethics education in Turkey}

Basic research, clinical research, preventive and social medicine, and routine clinical practice all have their own teaching methods. They have differ- ent ways of problem solving according to their specific subject matter and purpose. Örs emphasised different aspects of medical ethics, involving different degrees of technicality at different levels of medical education, from general undergraduate teaching to a highly specific and sophisticated branch of medical ethics such as deontological logic. Moral value issues which appear in animal research, with its aim of producing biomedical knowledge by exploiting certain species and "transferring" the results to the health problems of human beings, are appreciably different from those of the physician-patient relationship. Örs said that we should not of course lose sight of the fact that within the more comprehensive moral context of "respect for life", we would find certain basic similarities in the ethics of these subsets.'

What are the main characteristics of medical ethics education? In the light of "universality" versus "relativism", the problem is whether moral problems are to be considered from a universal viewpoint or from within a cultural context. Moral value problems within the medical context stem essentially from conflicts among individuals and/or groups of individuals, for example professionals and society, and interdisciplinary conflicts. These conflicts arise because people have different interests, value judgments, opinions, roles and beliefs. Ors stressed that as far as ethics as an academic discipline and its teaching are concerned, they are less under the influence of the sociocultural geography than morals, which may be regarded as the sum total of moral values that have developed within the social context. Common aspects of medical activity and education ensure that there will be less difference or variety in medical ethics and its teaching than for "culturally determined" moral differences within the general social context. If we accept our values as our idealisations, they are objectifiable or exteriorisable and also defensible on ethical grounds. In light of this, some points should be emphasised in medical ethics education. These points can be summarised as follows:

1. at every level of ethics, the methodology must be clarified;

2. students must be encouraged to express their moral views and opinions freely;

3. the reasons for their opinions should be explained, and

4. teachers should not hesitate to express their own ethical decision making process and its rationale. ${ }^{1}$ 
Arda and Örs stress that "moral sensitization and the development of ethical awareness or consciousness regarding the value problems arising in different aspects of medical activity" is their approach to teaching medical ethics in Turkey. ${ }^{2}$ If medical ethics is accepted as applied moral philosophy, this approach involves a critical conceptual analysis and discussion in the realm of moral value problems in medicine with a view to emotional awakening and rational understanding so that students may develop the interrelated abilities of grasping, thinking, discussing, and communicating effectively and in an academically satisfying way. Although medical ethics cannot be separated conceptually or practically from medical practice and training generally, it cannot be a discipline like molecular biology or the history of art. This has very important academic and pedagogical implications. For this reason, an "ethicist" cannot be a competent person in this field. Another important point is the open ended nature of moral discussions. There is an underlying ethical rationale to be defended within the acceptable limits of ethical thinking, and there is also an expectation that one will learn from others.

\section{Clinical research and some examples of legislation in the light of good clinical practice (GCP)}

In 1988 when a discussion began about "Noreum Oleander (NO) extract", introduced with the claim that it could cure many and various types of cancer, the public first recognised the concept of medical ethics and the need for ethics committees in Turkey. ${ }^{3}$ After this debate, an ethics committee was set up by the Ministry of Health and it has been working on drug research. ${ }^{46}$ There was regulation of this subject, namely The Amendment Relating to Drug Research, which was issued on 29th January 1993. Its purpose was to establish the principles, stages and quality of scientific research on human beings necessary for experimentation on recently discovered chemicals and biological substances. This research in the field of medicine and biology, covers materials used for diagnostic, therapeutic and/or preventive purposes. The regulation contains some points which are extremely important for drug development and research, for example, stages of the drug research; qualifications required of the researchers; the duties of the local and central ethics committees; qualifications for membership of these committees; the necessary procedures for working on new drugs; specifications for protocols of clinical research and the procedures for admission, and sanctions. This regulation is derived from the Helsinki Declaration $(1964,1975,1983,1989)$ and the Turkish Medical Deontology Regulation (1960) and states that the researchers must obey the rules of these texts.

After this regulation came a circular which the drug and pharmacy general directorate of the Ministry of Health issued on the 29th of December 1995. This circular stressed the importance of ethical principles and the obligation to maintain ethical rules at national and international levels. ${ }^{7}$ This circular also contains two guidelines: The Guideline of Good Clinical Practice (GGCP) and The Guideline of Good Laboratory Practice (GGLP).

The GGCP clarifies the following points:

* the basic principles of GCP;

* the main rationale of the research design;

$\star$ the protection of volunteers;

* the responsibilities and working methods of ethics committees;

* the informed consent of human subjects;

* the duties and responsibilities of supporters;

* the purposes and responsibilities of monitoring and reporting;

$\star$ the general qualifications and responsibilities of researchers;

$\star$ the statistical analysis;

* quality guarantee;

$\star$ auditing and

* finance, insurance and economic responsibilities to the subjects.

The GGLP clarifies some of these points and I shall not discuss them further.

\section{Clinical research and the function of ethics committees in Turkey}

In the light of The Guideline of Good Clinical Practice some recent legislation has been introduced, directed at the ethics committees which had been created in the medical faculties and state hospitals of Turkey. The ethics committees of the faculty of medicine of Hacettepe University and of Gülhane Military Medical Faculty were the first medical ethics committees (1986) in this country. These were ad hoc committees dealing with ethical issues in biomedical research

Research ethics committees, (RECs) are still developing in this country. In 1993 there were only eight RECs in Turkey, though 24 medical faculties exist. ${ }^{8}$ All of them have played a role as an advisory board. At this stage they began by controlling and, if needed, cancelling, medical research programmes. There are important differences in the structural and operational features of RECs. As a result of faulty policies, the number of medical faculties is increasing in Turkey. In 1997 there were $41 .^{9}$ In most of these new faculties there is no REC. There are also other deficiencies, such as laboratory facilities and library services etc.

On the other hand, one of the non-governmental organisations, the Turkish Medical Association, has had an ethics committee (ECTMA) since February 1994. It takes a multidisciplinary approach; the members of the committee are academicians from medicine, nursing, philosophy, medical sociology/ anthropology and law and are interested in "health and ethics". One of the aims of ECTMA is to develop solutions to medical ethics problems concerning specific issues, especially those raised by the Turkish Medical Association. The association's ethics committee has investigated and produced approximately twenty documents as guidance for physicians and one of these is dedicated to patient rights in medical research. ${ }^{10}$ 
A case discussion of respiratory distress syndrome (RDS): the experience of the research ethics committees

While informed consent is an extremely importan concept in routine medical practice, some of the medical ethics problems in underdeveloped countries are a result of the paternalistic character of the physician-patient relationship. For that reason, informed consent is not well known as a patient's right. In routine practice, the documented consent of the patient, especially for surgical operations, is accepted as necessary by physicians to provide the physicians with protection from the law.

Since the introduction of research ethics, researchers now recognise that the consent of human subjects is extremely important. But the necessary conditions for consent, the extent of the information that should be given, and method of giving information to subjects are not commonly known about and understood in Turkey. For instance, there are no compulsory clinical ethics lectures in this country, except at one medical school, Marmara University Medical School. We can also say that there is no formal and special ethics education for researchers. They obtain some communication skills by working with more experienced "seniors" in the team. This creates a random level of practice which depends on the sensitivity of the senior colleagues from whom they are learning.

Informed consent has also a very vital function in research involving vulnerable subjects such as children, prisoners, or people with mental disorders. Last year, research involving premature infants with respiratory distress syndrome (RDS) began a discussion in academic circles about research ethics in Turkey. There were four groups of infants with RDS. Three groups were given therapy including aminophylline, dexamethasone, aminophylline + dexamethasone, and there was one control group which received hospitalisation and oxygen, but no drug treatment. The findings of this "research" were published and the mortality rate was shown to be very high $(33 \%)$ for those infants in the control group who had had no medical treatment. ${ }^{11}$ This "research" was carried out in a very new university hospital, but it is not clear whether it had received the approval of the ethics committee; why the "research" was stopped when the deaths began in the control group; which criteria determined the contents of the groups, or whether the families had given informed consent.

\section{Conclusion}

Especially in developing societies, investigators have to recognise the different factors which are important in influencing and shaping people's behaviour. All reasonable efforts should be made to obtain informed consent from participants in studies and research teams should never initiate research on human subjects without obtaining each subject's informed consent unless they have received explicit approval to do so from a research ethics committee. Guidelines issued by CIOMS emphasised that investigators must convey information in a way that is appropriate to an individual's level of understanding and also that they must bear in mind that the ability to understand the information necessary to give informed consent depends upon the individual's maturity, intelligence, education, and rationality. The guidelines also suggest that the investigator might administer an oral or a written test to check whether the information has been adequately understood. ${ }^{12}$

As medical ethics is a new subject in Turkey, and for that reason is still not dealt with sufficiently in the media, adequate sensitivity and information have not so far been shown either by the medical profession or the public. People who suffer from diseases and are receiving health care and patients who volunteer for medical research, are generally not aware of their legal and/or "ethical" rights. Generally speaking, research groups are interested in the moral problems of their activity only when a warning or criticism has been directed at them. So, the evaluation criteria of the ethics committees are also important in maintaining ethical sensitivity in researchers. It has also been proposed that RECs can play a central educational role in helping physicians to be aware of moral problems and that they can contribute to the training of other medical doctors and colleagues. Thus the RECs could have a complementary function in respect of medical ethics education, which is deficient in many ways in this country. It must also be stressed that when undertaking research involving children or prisoners in underdeveloped countries great sensitivity and particular care are required. ${ }^{13}$

Berna Arda, MD, MedSpec, PhD, is Professor of Medicine, Department of Medical Ethics, Medical School, Ankara University, Ankara, Turkey.

\section{References}

1 Ors Y. Teaching medical ethics in the subjunctive mood. Bulletin of Medical Ethics 1993;93:31-6.

2 Arda B, Ors Y. Teaching medical ethics with an ethics to teach. Bulletin of Medical Ethics 1996;116:19-22.

3 Oğuz Y, Arda B. Bioethics in Turkey. Bulletin of Medical Ethics 1991;73:13-17.

4 Arda B, Sahinoglu PS. Bioethics in Turkey. Eubios fournal of Asian and International Bioethics 1995;5,3:64-5.

5 Arda B, Sahinoglu PS. Bioethics in a secular perspective. Eubios fournal of Asian and International Bioethics 1995;5,4:95-6.

6 Gournal of Asian and International Bioethics 1995;5,4:95-6. Törkey

Committes. Syndrome, 1994;6:84-7 (in 7 Kayaalp O. The essentials of the clinical pharmacology. Ankara: Tas Kitabevi, 1996: 79-90 (in Turkish).

8 Karakaya H, Oguz Y. Research Ethics Committees in Turkey. fournal of Ankara Medical Faculty 1993;46,2:321-6 (in Turkish)

9 Akaydın M, Aksakoğlu G, Arda B, Arığul O, Bulut A, Coskun $\mathrm{B}$, et al.Turkish Medical Association report on medical education. Ankara: Turkish Medical Association, 1997 (in Turkish)

10 Arda B. The activities of the ethics committee of the Turkish Medical Association. International fournal of Bioethics 1996;7,3: 235.

11 Kaya S, Dilmen U, Toppare M, Şenses D, Kitapçı F. Alternative approaches in the treatment of RDS. New fournal of Medicine 1996;13,6:370-2 (in Turkish).

12 Council for the International Organisation of Medical Sciences. International ethical guidelines for biomedical research Sciences. International ethical guidelines for biomed

13 Görkey S. Experimentations on children and ethical principles. Syndrome, 1994;6:82-5 (in Turkish). 